\title{
Technology of photocathode production
}

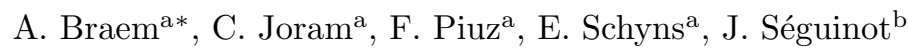 \\ ${ }^{a}$ CERN, EP Division, Geneva, Switzerland \\ ${ }^{\mathrm{b}}$ Collège de France, Paris, France
}

Thin film photocathodes have been developed both for gas and vacuum based photodetectors. Over the last years we have gained experience in fabricating large area reflective CsI and semi-transparent mono- and bialkali-cathodes, i.e. $\mathrm{Rb}_{2} \mathrm{Te}$ and $\mathrm{K}_{2} \mathrm{CsSb}$. Today we are producing stable cathodes with excellent quantum efficiency for large MWPC based pad detectors and Hybrid Photodiodes on a routine basis. We give an overview of the technologies involved in the preparation of the substrates, the cathode processing and the encapsulation. The dedicated processing plants and instrumentation, required for monitoring and characterisation, is discussed.

Keywords: RICH; Photodetector; Photocathode; Cesium Iodide; CsI, Rubidium Telluride; Bialkali; ITO; Hybrid Photodiode; HPD;

\section{Introduction}

While the first generation of large RICH detectors like DELPHI and SLD CRID relied on gaseous photoconverters, most of the current and future Cherenkov counters exploit the numerous advantages of solid photocathodes. Still, the requirements have not changed much since then: The RICH technique demands in general large photosensitive surfaces $\left(\sim \mathrm{m}^{2}\right)$, where high sensitivity, fine pixelization $\left(\leq \mathrm{cm}^{2}\right)$, stability and cost effectiveness are the most important requirements. In the following we report about two different technologies which have been developed for these applications.

Reflective CsI photocathodes allow to build very large and thin gas based photodetectors which are cost effective and can be operated inside magnetic fields. Their sensitivity is however limited to the VUV part of the Cherenkov spectrum $\left(\mathrm{E}_{\gamma} \geq 6.2 \mathrm{eV}\right)$. The use of semi-transparent alkali photocathodes, sensitive to visible and UV light, have considerably increased the potential of RICH counters. However, these flimsy layers react with almost any substance hence they have to be sealed in a vacuum envelope to preserve their high sensitivity. In both cases large dedi-

\footnotetext{
*Corresponding author, Andre.Braem@cern.ch
}

cated coating facilities are needed to produce the photocathodes and assess their quality.

\section{Reflective CsI photocathodes for gas based detectors}

Reflective CsI photocathodes for photon detection with gas based detectors have been studied since the late 1980s $[1,2]$. Large area CsI photocathodes for RICH detectors $\left(\sim 0.25 \mathrm{~m}^{2}\right)$ were developed in the early 1990s by the CERN RD26 collaboration [3]. Today, CsI photodetection planes are widely utilised in RICH detectors [4], consisting of arrangements of photocathodes of up to $60 \times 60 \mathrm{~cm}^{2}$ size, e.g. Ref. [5].

The key requirements for CsI photocathodes are high quantum efficiency (QE) and uniform and stable response above the threshold $6.2 \mathrm{eV}$. CsI is known to be hygroscopic hence CsI photocathodes must be transferred in a humidity free environment. The QE depends on the thickness of the CsI film. A thickness of $\sim 60 \mathrm{~nm}$ yields a good QE, i.e. photoelectrons created at a depth $>60 \mathrm{~nm}$ have a negligible escape probability [6]. Thicker CsI films have no higher QE, however they possess an increased robustness with respect to low level moisture absorption and provide a more uniform film distribution over the substrate. All our standard photocathodes have a CsI film 


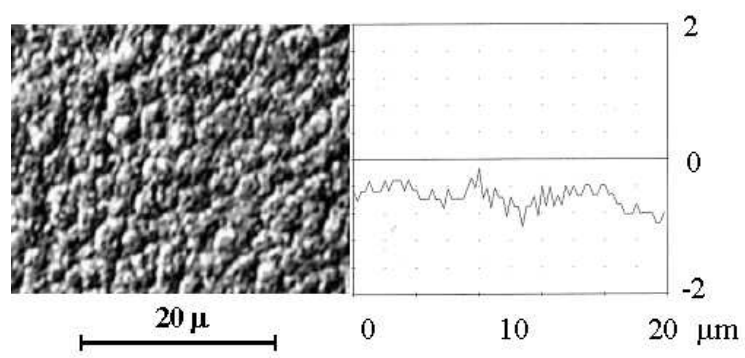

Figure 1. Microscopic view and profilometric measurement of a typical pad substrate.

thickness of $300 \mathrm{~nm}$.

\subsection{Substrate preparation for CsI photo- cathodes}

New standards in electroplating technology allow the production of large $\left(\sim 30 \times 40 \mathrm{~cm}^{2}\right)$ multi-layer printed circuit boards (PCB's) providing a leaktight photocathode surface with gold plated nickel layers on $\sim 8 \times 8 \mathrm{~mm}^{2}$ copper pads. The roughness of a $\mathrm{Au} / \mathrm{Ni} / \mathrm{Cu}$ pad substrate is shown in figure 1 , exhibiting microscopic variations of the surface structure of the order of $0.2 \mu \mathrm{m}$. We tested substrates of different surface roughness and found no significant impact on the QE performance [7]. However, ultrasonic cleaning prior to the installation of the photocathode in the deposition plant, using strong detergents, de-mineralised water and pure alcohol, has been found as crucial. Nevertheless, XPS surface analyses have shown that a $\sim 1 \mathrm{~nm}$ carbon layer from residual atmospheric contaminants remains present on the substrate before the CsI coating $[8]$.

\subsection{The CsI vacuum evaporation process}

A dedicated vacuum evaporation plant has been constructed at CERN for the coating of large area (up to $60 \times 60 \mathrm{~cm}^{2}$ ) cathodes [9]. The PCB substrate is horizontally mounted above four premelted CsI sources located at a fixed distance from the corners which allows to achieve a uniform CsI film thickness within $\sim 5 \%$. We typically foresee 48 hours pumping time to obtain a vacuum level below $10^{-4} \mathrm{~Pa}$. Due to the material composition of the $\mathrm{PCB}$ we have chosen to limit vacuum bakeout temperatures to $60^{\circ} \mathrm{C}$ according to previous measurements. Residual gas analyses prior to the evaporation normally show high concentrations of water and hydrocarbons i.e. $\sim 40 \% \mathrm{H}_{2} \mathrm{O}$ and $\sim 40 \% \mathrm{C}_{\mathrm{x}} \mathrm{H}_{\mathrm{y}}$ at a pressure of $\sim 4.10^{-5} \mathrm{~Pa}$. The four sources are powered in series and heated by Joule effect to evaporate a $300 \mathrm{~nm}$ CsI film at a monitored low rate of $\sim 1$ $2 \mathrm{~nm} / \mathrm{sec}$ to minimise the probability of CsI dissociation. After the CsI coating, the photocathodes are kept 8 hours under vacuum at $60^{\circ} \mathrm{C}$ [10]. Before re-opening the deposition plant, the CsI photocathode is encapsulated in a leaktight transferbox under dry argon. For future photocathode series productions, our evaporation plant is being upgraded with a 'VUV scanner system' for in situ quality evaluation over the full cathode surface.

\subsection{The QE performance of CsI photo- cathodes}

The performance of the full scale prototype photocathodes we produce for the ALICE/HMPID [9] is investigated in a CERN testbeam facility. The QE is evaluated with a RICH detector using single photon counting and full detector simulation [11]. Figure 2 shows the QE results obtained for six recently produced prototype photocathodes (PC33-PC38) of which five have the same QE within the uncertainty of $\sim 5 \%$. The initial spectral response of PC35 and PC36 turned out to be very low. The substrates were possibly polluted prior to the CsI coating. After thorough cleaning, PC35 was given a new CsI coating and was re-tested to perform very well (PC35 fig. 2).

Earlier produced photocathodes have been used for ageing and long term stability studies. During operation, the detector gas is monitored to contain $<10 \mathrm{ppm} \mathrm{O}_{2}$ and $<10 \mathrm{ppm}_{2} \mathrm{O}$. Beam tests before and after exposure to higher levels of oxygen (up to $100000 \mathrm{ppm}$ ) and water vapours (up to $40 \mathrm{ppm}$ ) did not show any effect on the photocathode performance [12]. Moreover, no degradation of the quantum efficiency has been observed on photocathodes which were periodically exposed to testbeams, corresponding to local integrated charge densities of $1 \mathrm{mC} / \mathrm{cm}^{2}$ [13]. 


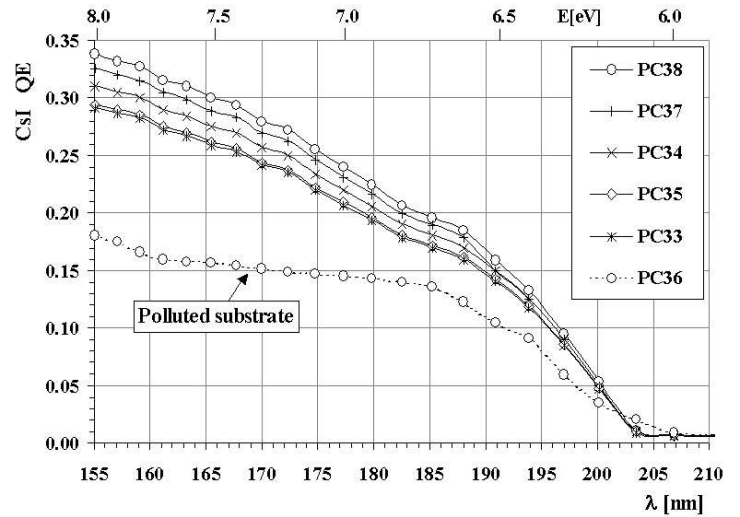

Figure 2. Quantum efficiency of 6 most recent CsI photocathodes produced (for clarity, the $\sim 5 \%$ uncertainty bars have been omitted).

\section{Semi-transparent photocathodes for vacuum based photodetectors}

Alkali antimonide and telluride photocathodes have a long history in vacuum photo tubes and image intensifiers. The book by Sommer [14], still the standard reference in the field, describes basic recipes for the cathode fabrication. The appealing feature of this cathode family is their high sensitivity which can extend from the near IR to the far UV. The maximum quantum efficiency is limited to about $25-30 \%$ for semi-transparent cathodes and may reach $40 \%$ for reflective ones.

The cathode thickness plays a crucial role for the sensitivity and spectral response of semitransparent cathodes. The conflicting requirements of total photon absorption and efficient electron escape lead to cathodes with a thickness of a few tens of nm. Also the stoichiometry and the effectiveness of the chemical reaction of the various evaporated components, e.g. K, Cs, and $\mathrm{Sb}$ in case of a bialkali cathode, are of importance. Optimal results can only be reached if the response of the cathode is monitored throughout the whole process.

During the last years a UHV evaporation facility has been built at CERN, with the original aim to develop large Hybrid Photodiodes (HPD) [15] for the readout of the LHCb RICH counters [16]. It consists of a large vacuum tank, equipped with a powerful UHV pumping and a custom designed bake-out system. End pressures below $10^{-7} \mathrm{~Pa}$ and low water partial pressures are reached. It is equipped with a press mechanism, which allows to seal tubes by means of a cold indium technique. A calibrated quantum efficiency monitoring system completes the set-up.

\subsection{The fabrication of the Pad HPD}

The Pad HPD is a round HPD of 5 inch diameter (see figure 3 ). Its main features and performance are described in $[17,18]$.

The cathode of the Pad HPD is fabricated in an external process to minimise both the pollution with alkali metals and the thermal load of the silicon sensor. Once the photocathode is vacuum deposited through the bottom opening of the glass envelope, the tube is sealed off in situ by the base plate, on which the silicon sensor and its electronics have been pre-mounted.

Crucial for the success are the numerous preparatory steps which the envelope and the baseplate undergo prior to installation in the vacuum tank: polishing of metal components (electrodes, skirt) for optimum HV stability; polishing of entrance window; deposition of $\mathrm{Ni}$ and $\mathrm{Au}$ inter-diffusion layers on the indium; sealing surfaces; pre-melting of In in the V-shaped groove on the baseplate; deposition of a $\mathrm{Cr}$ peripheral ring on entrance window; application of conductive glue contacts between skirt and Cr ring.

The modular design of the HPD allows recycling of all its components. In case components are to be recycled from a previous processing, metal and glass surfaces are exposed to different sequences of acids in order to chemically etch away any remaining traces before the above procedure. Only after a final cleaning procedure in a ultra sonic bath (teepol detergent/water/distilled water/alcohol) and subsequent drying the components are installed in the vacuum tank.

\subsection{The bi-alkali antimonide process}

For bi-alkali $\mathrm{K}_{2} \mathrm{CsSb}$ photocathodes we have adopted and optimised a so-called co-evaporation process. After an extended bakeout (48-72 hours) of the complete plant at $160^{\circ} \mathrm{C}$ and of the glass envelope at $300^{\circ} \mathrm{C}$ (internal heating element), the 
envelope is maintained at $150^{\circ} \mathrm{C}$ for the complete deposition. The evaporation sources are positioned close to the centre of curvature of the HPD entrance window. First potassium is evaporated from a commercial dispenser, producing a film over the full glass substrate which leads to a small but stable photocurrent. Then $\mathrm{Sb}$ is co-evaporated from a source, which consists of a small Ta boat, on which a Sb bead has been premelted under vacuum. The simultaneous evaporation of $\mathrm{K}$ and $\mathrm{Sb}$ is continued until a peak of the sensitivity for white light is observed. At this stage the formed $\mathrm{K}_{3} \mathrm{Sb}$ has a quantum efficiency at $400 \mathrm{~nm}$ of typically $3-4 \%$. The cathode is then gradually activated by $\mathrm{Cs}$ to form $\mathrm{K}_{2} \mathrm{CsSb}$,

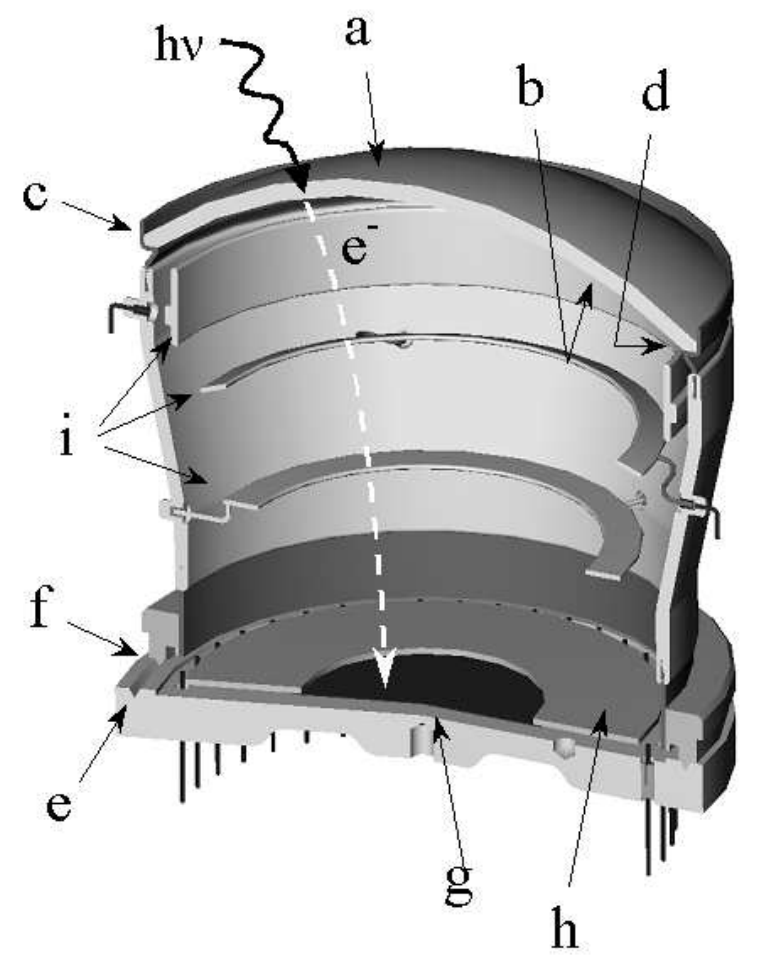

Figure 3. 3D view of the Pad HPD. The individual components are: $\mathrm{a}=$ entrance window, $\mathrm{b}=$ photocathode, $\mathrm{c}=$ skirt, $\mathrm{d}=$ peripheral $\mathrm{Cr}$ ring, $\mathrm{e}=$ groove, $\mathrm{f}=$ knife edge, $\mathrm{g}=$ silicon sensor, $\mathrm{h}$ $=$ ceramic $\mathrm{PCB}+$ electronics, $\mathrm{i}=$ focusing electrodes. marked by a more or less pronounced maximum of the sensitivity. The above steps and/or alternating $\mathrm{Sb}-\mathrm{Cs}$ evaporations are repeated until the optimum spectral response is obtained.

\subsection{The rubidium telluride process}

Also our $\mathrm{Rb}_{2} \mathrm{Te}$ process [17] is based on the co-evaporation technique, however the substrate temperature is lower $\left(70^{\circ} \mathrm{C}\right)$ and the photocurrent is monitored for monochromatic illumination at $250 \mathrm{~nm}$. We start with Rb (commercial dispenser) and then co-evaporate Te (pre-melted on a Mo boat) until peak efficiency is reached.

\subsection{ITO as transparent conductive under- layer}

Semi-transparent solar blind photocathodes, like $\mathrm{Cs}_{2} \mathrm{Te}$ or $\mathrm{Rb}_{2} \mathrm{Te}$, have the disadvantage of a high surface resistivity (in excess of $10^{10} \Omega$ /square). For cathodes of a certain diameter usually very thin metallic underlayers, e.g. $\mathrm{Cr}$, are pre-deposited on the glass substrate to allow a sizeable photocurrent and a uniform electric field distribution over the full cathode surface. A drawback of this method is the transmission loss associated with the metallic underlayer. We have systematically studied thin conductive layers of ITO, a mixture of indium oxide and tin oxide, in comparison with chromium. Compressed pellets of ITO powder are evaporated by means of an electron gun. A post-treatment in an oven at $300^{\circ} \mathrm{C}$ in air for 8 hours re-oxidises the partially reduced compounds, decreases the resistivity and increases the transparency of the film. Figure 4 shows the transmission, averaged between $\lambda=$ 200 and $400 \mathrm{~nm}$ versus the surface resistivity of thin $(1.6-6.3 \mathrm{~nm})$ ITO and Cr films, deposited on quartz substrates. At the same resistivity ITO films exhibit a significantly higher transparency. Resistivity values around $1 \mathrm{M} \Omega$ /square, which are considered as sufficient for our application, can be obtained with ITO at an average transmission loss of only $\sim 15 \%$, compared to $\sim 35 \%$ for $\mathrm{Cr}$. Figure 5 shows the difference in spectral transmission of the UV extended glass window before and after coating with a $3.2 \mathrm{~nm}$ thick ITO film. The ratio of the two curves exhibits the increasing absorption of ITO below $200 \mathrm{~nm}$. 


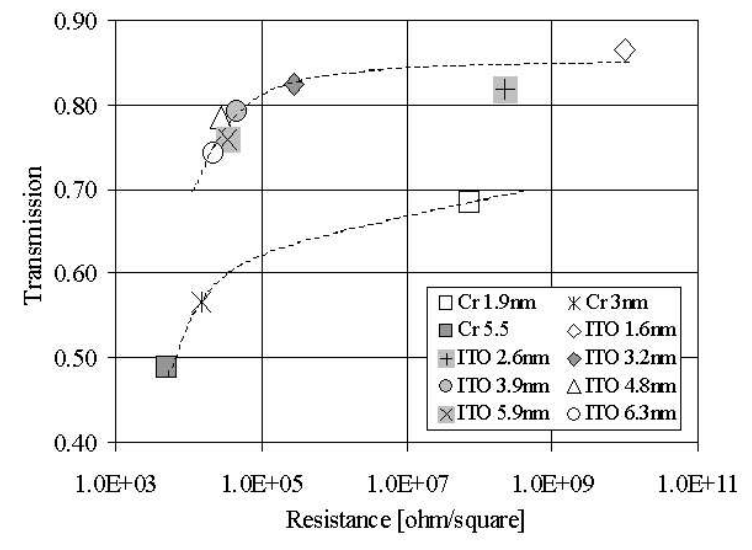

Figure 4. Measured surface resistance versus average transmission (200-400 $\mathrm{nm}$ ) of ITO and $\mathrm{Cr}$ thin films of various thickness on quartz substrates.

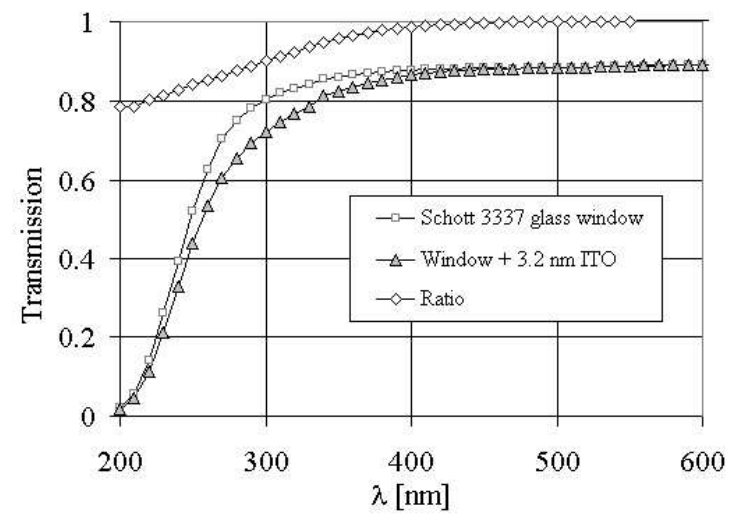

Figure 5. Transmission of UV extended glass with and without ITO coating.

\subsection{The performance of $\mathrm{K}_{2} \mathrm{CsSb}$ and $\mathrm{Rb}_{2} \mathrm{Te}$ photocathodes}

The bi-alkali $\mathrm{K}_{2} \mathrm{CsSb}$ process, involving $3 \mathrm{com}$ ponents and sometimes up to 20 steps, required a relatively long optimisation. Quantum efficiency peak values in the range of 22 to $28 \%$ are now routinely reached (see fig. 6). The cathode yields are measured to be stable and have a radial uniformity of $\pm 10 \%$. The uniformity measurement is slightly biased due to some none-absorbed incident photons reflected back onto the cathode by the $\mathrm{Si}$ sensor. The mono-alkali $\mathrm{Rb}_{2} \mathrm{Te}$ process is simpler and very robust. All cathodes grown on

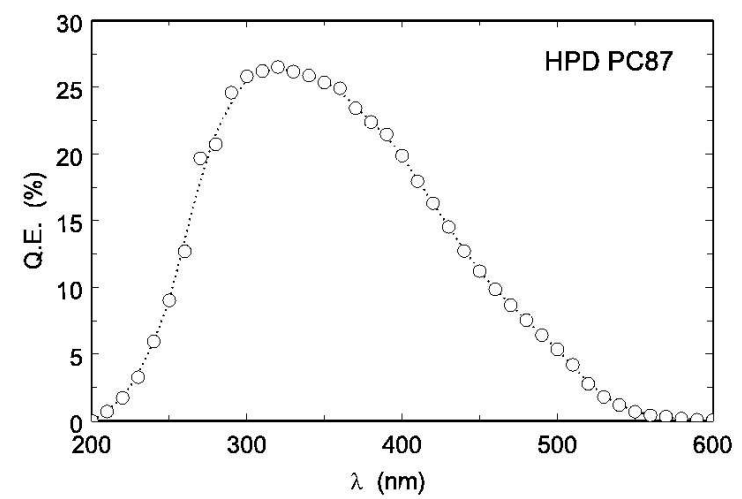

Figure 6. Quantum efficiency of a $\mathrm{K}_{2} \mathrm{CsSb}$ photocathode produced on a UV extended HPD glass window.

ITO underlayers showed QE values of $\approx 15-18 \%$ at $\lambda \leq 250 \mathrm{~nm}$ (see figure 7 ). Efficiencies below $1 \%$ at $300 \mathrm{~nm}$ and less than $0.01 \%$ at $400 \mathrm{~nm}$ were measured. The peak QE significantly exceeds values quoted by commercial phototube suppliers. $\mathrm{Rb}_{2} \mathrm{Te}$, directly evaporated on glass, gave low and with time degrading results.

\section{Summary}

The continuous effort of all involved persons and the permanent optimisation of the processes was necessary to arrive at mature photocathode technologies with reproducible results. The reflective VUV CsI photocathode is less demanding as far as the processing is concerned, however, after the vacuum deposition the cathode has to be kept in a well controlled gas atmosphere throughout its full life cycle. This poses constraints on detector assembly, operation and maintenance. Vacuum based photodetectors require a higher technological effort during component preparation, processing and sealing. The sealed detector is a robust device, which can then be handled and operated in normal atmosphere. Common to both approaches is the need of several sophisticated and well mastered technologies, like UHV, galvanic deposition, in situ vacuum sealing and encapsulated electronics. 


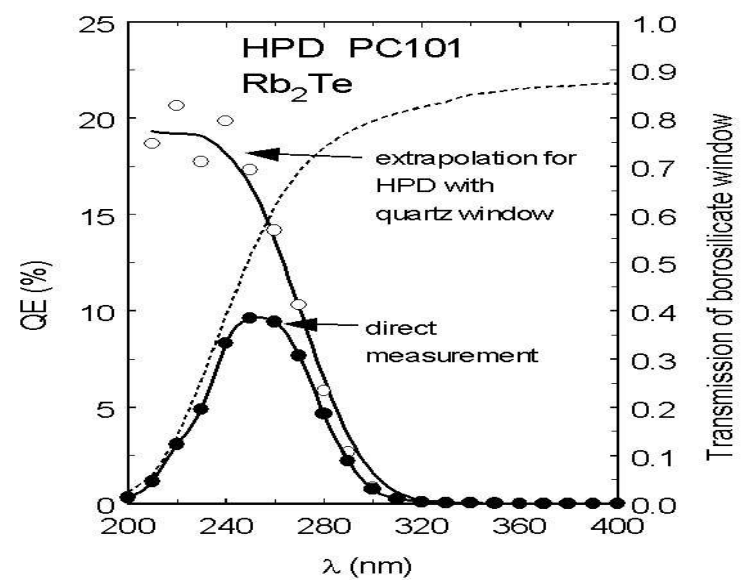

Figure 7. Quantum efficiency of an HPD with $\mathrm{Rb}_{2} \mathrm{Te}$ photocathode. The transmission of the HPD borosilicate glass window (right y-scale) suppresses the QE for $\lambda \leq 300 \mathrm{~nm}$ (labelled "direct measurement"). Therefore the extrapolated QE for an HPD with quartz window is also shown.

\section{Acknowledgements}

The development and production of the described photocathodes would not have been possible without the very competent and reliable support of our technical staff at CERN. We would like to thank D. Carminati, C. David, R. Dye, L. Kottelat, M. Malabaila and M. Thiebert for excellent services. The stimulating and fruitful collaboration with the experiments ALICE, COMPASS, CLUE, LHCb and RD26 has originally initiated and continuously promoted these developments. The open exchange of technical information with the Weizmann Institute of Science, Rehovot, Israel (A. Breskin et al.) and the Technical University Munich (J. Friese et al.) was greatly appreciated.

\section{REFERENCES}

1. J. Séguinot et al., Nucl. Instr. Meth. A 297 (1990) 133.

2. A. Breskin, Nucl. Instr. Meth. A 371 (1996) 116.

3. CERN-RD26: CERN/DRDC 92-3, CERN/DRDC 96-20.
4. E. Nappi, Nucl. Instr. Meth. A 471 (2001) 18.

5. The COMPASS Experiment, CERN/SPSLC 96-14,

G. Baum et al., Nucl. Instr. Meth. A 433 (1999) 207.

6. T. Boutboul et al., J.Appl.Phys, 84 (1998) 2890.

7. E. Schyns, Nucl. Instr. Meth. A 494 (2002) 441.

8. M. Davenport, M. Taborelli, private communications.

9. ALICE collaboration, HMPID Technical Design Report, CERN/LHC 98-19, p116 - 120, http://alice-hmpid.web.cern.ch/alice-hmpid.

10. D. Anderson et al., Nucl. Instr. Meth. A 326 (1993) 611,

A. Buzulutskov et al., Nucl. Instr. Meth. A 366 (1995) 383.

11. A. Di Mauro et al., Nucl. Instr. Meth. A 433 (1999) 190

F. Piuz et al., Nucl. Instr. Meth. A 433 (1999) 178.

12. A. Di Mauro et al., Nucl. Instr. Meth. A 461 (2001) 584.

13. A. Di Mauro et al., 'Aging of large area CsI photocathodes for the ALICE HMPID prototypes', contribution to the International Workshop on Aging Phenomena in Gaseous Detectors, Hamburg, 2-5 October 2001, submitted to Nucl. Instr. and Meth..

14. A.H. Sommer, Photoemissive materials, E. Krieger, New York, 2nd edition, 1980.

15. C. Joram, Nucl. Phys. B (Proc. Suppl.) 78 (1999) 407.

16. LHCb Technical Proposal. CERN/LHCC 984, $\mathrm{LHCC} / \mathrm{P} 4$.

17. A. Braem et al., Nucl. Instr. Meth. A 442 (2000) 128.

18. A. Braem et al., Nucl. Instr. Meth. A 478 (2002) 400. 